\title{
A Convolutional Neural Network Approach to Thickness Determination using Position Averaged Convergent Beam Electron Diffraction
}

\author{
Weizong $\mathrm{Xu}^{1}$ and James M. LeBeau ${ }^{1}$ \\ 1. Department of Materials Science and Engineering, North Carolina State University, Raleigh, NC, \\ USA
}

Position averaged convergent beam electron diffraction (PACBED) can accurately measure local specimen thickness with nanometer resolution, which is critical in quantitative scanning transmission electron microscopy (STEM) [1]. These thickness measurements are conducted by pattern matching experiment to simulations, either by least squares fitting (LSF) or by eye. This process, however, can be slow and inaccurate, since only slight contrast feature changes are used be distinguish patterns that are within a few $\mathrm{nm}$. In recent years, convolutional neural networks (CNN) have shown excellent performance in tasks such as self-driving cars, face detection, and text recognition. With multiple convolutional layers in deep, $\mathrm{CNN}$ can automatically extract the local features from the images without any feature engineering procedures. The above advantage suggests the promising $\mathrm{CNN}$ application in determining thickness from PACBED with different image contrast and patterns.

In this study, we seek a deep convolutional neural network approach to determine the local specimen thickness from PACBED with $1 \mathrm{~nm}$ resolution from $1 \mathrm{~nm}$ to $120 \mathrm{~nm}$. Starting from a pre-trained AlexNet network[2], we transfer its network architecture to learn this new task[3]. Specifically, we keep the weights of convolutional and first two fully connected layers from the original AlexNet and replace the last fully-connected and softmax layer for 120 categories as illustrated in Figure 1. The weights in the network are fine-tuned in subsequent training by continuing the backpropagation with a 10 times faster learning rate in the last fully-connected layer. The total training process is conducted using about 5.8 million images, which are random affine transformed, brightness adjusted, rotated, and noise added from the simulated PACBED patterns in different thickness. To reduce the overfitting, dropout is applied in the fully connected layers with a ratio of $0.5[4]$. The trained network shows $99.1 \%$ accuracy from the testing data (based on simulated PACBED patterns). The approach achieves an overall $85 \%$ accuracy when comparing experimentally acquired PACBED patterns, where the thickness determination error is within 1-2 nm, suggesting a good generalization of the neural network for realworld applications.

Figure 2a shows the experimental PACBED pattern of a $<100>$ orientated $\mathrm{SrTiO}_{3}$ crystal. The local bright/dark patterns and contours from the PACBED are activated in the fine-tuned CNN in the convolutional layers, indicating the success of the $\mathrm{CNN}$ to subtract regional features from PACBED patterns. The thickness determined from this PACBED by the CNN is $51 \mathrm{~nm}$, compared to $51-52 \mathrm{~nm}$ by visual inspection. For a more faithful comparison, the thickness is further determined from least square fitting (LSF), which searches the PACBED pattern for minimum intensity deviation between simulation and experiment. Consistent results are found among these methods. For wider thickness range, CNN can provide reliable measurements as demonstrated in Figure $2 \mathrm{~b}$. In particular, it can accurately determine the thickness when the sample is less than 6-8 nm thick, where the LSF method becomes inaccurate. We also notice a larger deviation from experimental and simulated PACBED patterns at thick regions above $70 \mathrm{~nm}$ due to the diffuse electron scattering, which also degrades LSF accuracy. A uniform background substation on the PACBED image is made to improve the reliability of both the LSF and CNN analysis. 
Moreover, after training the CNN approach is very fast, with average identification achieved within $0.001 \mathrm{~s}$ per image, which largely outperforms the LSF. These speeds open the possibility to efficiently determining local thickness or structural changes during STEM imaging [5].

\section{References:}

[1] J LeBeau et al, Ultramicroscopy 110 (2010) p. 118.

[2] A Krizhevsky, I Sutskever and G Hinton, Advances in Neural Information Processing Systems 25 (2012) p.1097.

[3] S Pan and Q Yang, IEEE Transactions on Knowledge and Data Engineering 22 (2010) p.1345.

[4] N Srivastava et al, Journal of Machine Learning Research 15 (2014) p.1929.

[5] The authors acknowledge the support from the National Science Foundation (NSF) (DMR-1350273, DMR-1151568) and Air Force Office of Scientific Research (FA9550-14-1-0182). We thank analytical Instrumentation Facility (AIF) at North Carolina State University supported by the State of North Carolina, the National Science Foundation (ECCS-1542015) and the North Carolina Research Triangle Nanotechnology Network from the National Nanotechnology Coordinated Infrastructure.

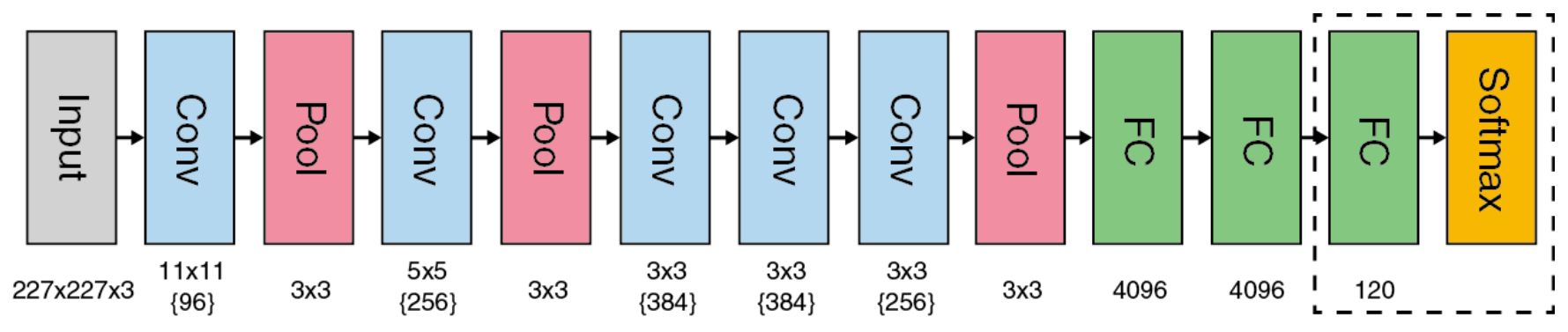

Convolutional+ReLU Layer

Max Pooling Layer

Fully Connected Layer

Figure 1. Illustration of the convolutional neural network architecture in this work.
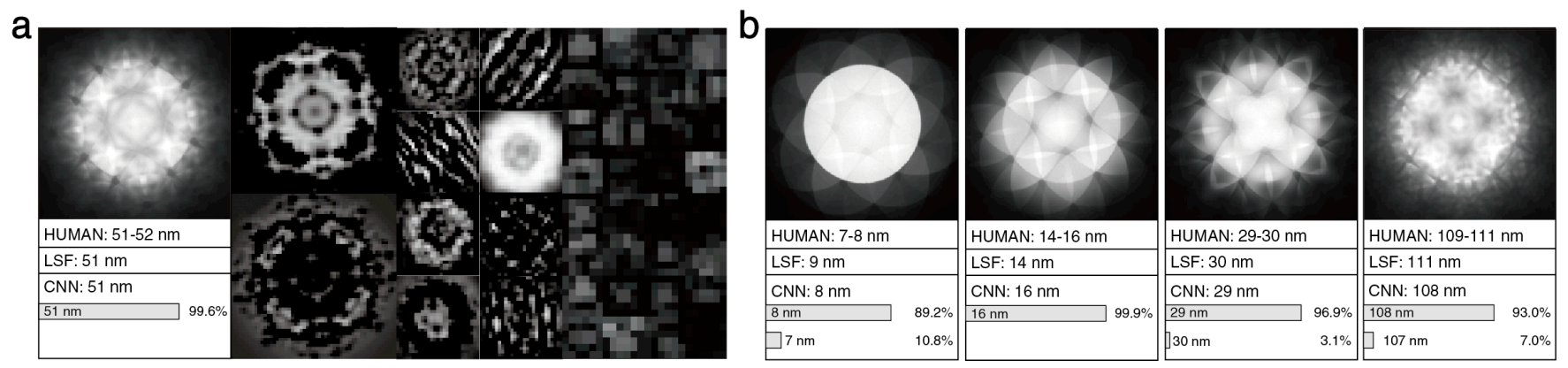

Conv 1

Conv 2

Conv 5

Figure 2. Example of experimental PACBEDs and their thickness identification by human, least square fitting (LSF) and convolutional neural network (CNN). Selected network activations in the first, second and fifth convolutional layers after ReLU are illustrated in (a). 